\title{
Active Directory Field Guide
}

Laura E. Hunter 


\section{Active Directory Field Guide}

\section{Copyright $\odot 2005$ by Laura E. Hunter}

All rights reserved. No part of this work may be reproduced or transmitted in any form or by any means, electronic or mechanical, including photocopying, recording, or by any information storage or retrieval system, without the prior written permission of the copyright owner and the publisher.

ISBN (pbk): 1-59059-492-4

Printed and bound in the United States of America 987654321

Trademarked names may appear in this book. Rather than use a trademark symbol with every occurrence of a trademarked name, we use the names only in an editorial fashion and to the benefit of the trademark owner, with no intention of infringement of the trademark.

Lead Editor: Jim Sumser

Technical Reviewer: Alexander N. Nepomnjashiy

Editorial Board: Steve Anglin, Dan Appleman, Ewan Buckingham, Gary Cornell, Tony Davis, Jason Gilmore, Jonathan Hassell, Chris Mills, Dominic Shakeshaft, Jim Sumser

Assistant Publisher: Grace Wong

Project Manager: Beckie Stones

Copy Manager: Nicole LeClerc

Copy Editor: Ami Knox

Production Manager: Kari Brooks-Copony

Production Editor: Ellie Fountain

Compositor: Diana Van Winkle

Proofreader: Linda Marousek

Indexer: Kevin Broccoli

Artist: Diana Van Winkle

Cover Designer: Kurt Krames

Manufacturing Manager: Tom Debolski

Distributed to the book trade in the United States by Springer-Verlag New York, Inc., 233 Spring Street, 6th Floor, New York, NY 10013, and outside the United States by Springer-Verlag GmbH \& Co. KG, Tiergartenstr. 17, 69112 Heidelberg, Germany.

In the United States: phone 1-800-SPRINGER, fax 201-348-4505, e-mail orders@springer-ny.com, or visit http://www.springer-ny.com. Outside the United States: fax +496221 345229, e-mail orders@springer.de, or visit http://www.springer.de.

For information on translations, please contact Apress directly at 2560 Ninth Street, Suite 219, Berkeley, CA 94710. Phone 510-549-5930, fax 510-549-5939, e-mail info@apress.com, or visit http://www.apress.com.

The information in this book is distributed on an "as is" basis, without warranty. Although every precaution has been taken in the preparation of this work, neither the author(s) nor Apress shall have any liability to any person or entity with respect to any loss or damage caused or alleged to be caused directly or indirectly by the information contained in this work. 
For Mom, Dad, and Bryan 


\section{Contents at a Glance}

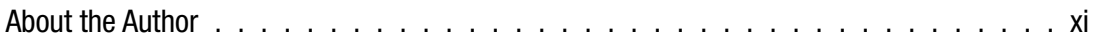

About the Technical Reviewer . . . . . . . . . . . . . . . . . . . xiii

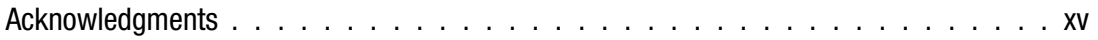

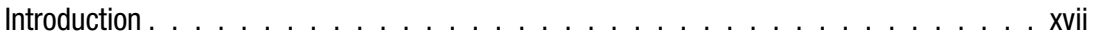

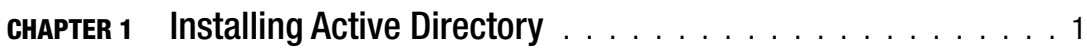

CHAPTER 2 Integrating the Network Infrastructure . . . . . . . . 39

CHAPTER 3 Daily Administration . . . . . . . . . . . . . . . . . . 77

CHAPTER 4 Deploying Group Policy . . . . . . . . . . . . . . . 113

CHAPTER 5 Active Directory Security . . . . . . . . . . . . . . . 149

CHAPTER 6 Managing Large-Scale Deployments . . . . . . . . . . 187

CHAPTER 7 Active Directory Migrations . . . . . . . . . . . . . . . 219

CHAPTER 8 Disaster Recovery . . . . . . . . . . . . . . . . . . . 251

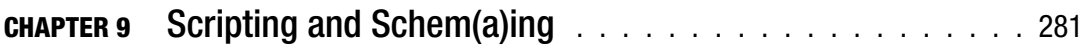

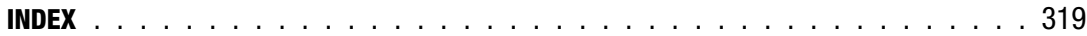




\section{Contents}

About the Author . . . . . . . . . . . . . . . . . . . . xi

About the Technical Reviewer . . . . . . . . . . . . . . . . . . . xiii

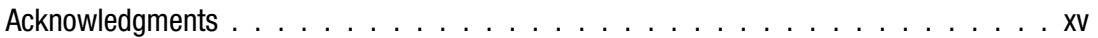

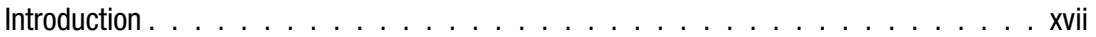

CHAPTER 1 Installing Active Directory $\ldots \ldots \ldots \ldots \ldots$

Planning Your Active Directory Installation . . . . . . . . . . . . . . 2

Creating the Logical Structure . . . . . . . . . . . . . . . 2

Creating the Physical Design . . . . . . . . . . . . . . . . 12

Installing Active Directory . . . . . . . . . . . . . . . . . . . . 20

Automating the Installation Process $\ldots \ldots \ldots . .24$

Scripting the AD Installation $\ldots \ldots \ldots . . \ldots 24$

Scripting a Bare Metal Install . . . . . . . . . . . . . . . . . . . . 29

Troubleshooting Active Directory Installations . . . . . . . . . . . . . 31

Troubleshooting Network Connectivity . . . . . . . . . . . . 31

Troubleshooting Name Resolution . . . . . . . . . . . . . . . . . 33

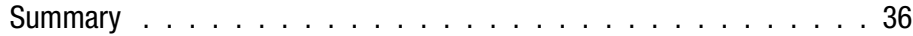

Additional Resources . . . . . . . . . . . . . . 37

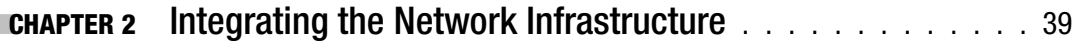

Deploying DNS . . . . . . . . . . . . . . . . . 40 40

Creating the DNS Infrastructure . . . . . . . . . . . . . . . . . 44

Configuring Forwarders . . . . . . . . . . . . . . . . 49

Securing DNS . . . . . . . . . . . . . . . .52

Integrating with Third-Party DNS . . . . . . . . . . . 56

Using DHCP . . . . . . . . . . . . . . . . . . . . 57

Installing $\mathrm{DHCP} \ldots \ldots \ldots \ldots$

Configuring DHCP Scopes $\ldots \ldots \ldots$

Integrating DHCP with DNS . . . . . . . . . . . . . . . . . 66

Supporting WINS and NetBIOS . . . . . . . . . . . . . . . . 68

Installing a WINS Server . . . . . . . . . . . . . . . . . . . . . . . . 69

Specifying Client Options for WINS $\ldots \ldots \ldots .71$

Configuring WINS Replication . . . . . . . . . . . . . . 72

Summary . . . . . . . . . . . . . . . . . . . . . . 75

Additional Resources . . . . . . . . . . . . . . . . . . .76 


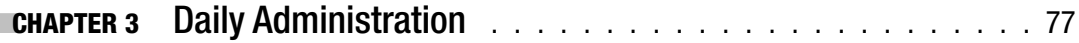

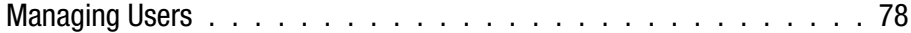

Creating Users . . . . . . . . . . . . . . . . . . . . . . . . . . 78

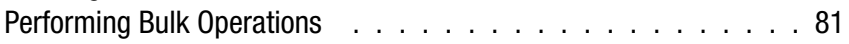

Configuring User Accounts . . . . . . . . . . . . . . . . . . 84 84

Delegating Administrative Authority . . . . . . . . . . . . . . 92

Managing Groups . . . . . . . . . . . . . . . . . . . . . . 94

Configuring Groups . . . . . . . . . . . . . . . . . . . . 95

Leveraging Group Nesting . . . . . . . . . . . . . . . 97

Creating Groups . . . . . . . . . . . . . . . . . . 99

Managing User Files . . . . . . . . . . . . . . . . . . 100

Configuring User Profiles . . . . . . . . . . . . . . . . 100

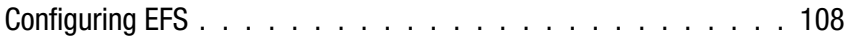

Recovering Encrypted Files . . . . . . . . . . . . . . . 109

Summary . . . . . . . . . . . . . . . . . . . . . . . 111

Additional Resources . . . . . . . . . . . . . . . . . . . . . . . 111

ChAPTER 4 Deploying Group Policy . . . . . . . . . . . . . . . . . . . 113

Group Policy Management Console . . . . . . . . . . . . . . . . . . . 114

Migrating Group Policy Settings . . . . . . . . . . . . . . . . 117

Modeling Group Policy . . . . . . . . . . . . . . . . . . . . 119

Monitoring Group Policy Results . . . . . . . . . . . . . 120

Who Gets What? Deploying Group Policies . . . . . . . . . . . . 120

Using Organizational Units . . . . . . . . . . . . . . . . . . 120

Configuring Policy Inheritance . . . . . . . . . . . . . . . . 121

Customizing Policy Inheritance . . . . . . . . . . . . . . . . . 122

Controlling the Desktop . . . . . . . . . . . . . . . . . . . . . 125

Configuring Lockdown (Kiosk) Workstations . . . . . . . . . . 126

Using Software Restriction Policies . . . . . . . . . . . . . . 127

Securing Client Operating Systems $\ldots \ldots 134$

Configuring Software Deployment . . . . . . . . . . . . . . . . 141

Creating an Installation Package . . . . . . . . . . . . . . . . . 141

Understanding Deployment Options . . . . . . . . . . . . . . 142

Using Advanced Techniques . . . . . . . . . . . . . . . . . . . . 145

Controlling the Registry and File System . . . . . . . . . . . . . 146

Using Restricted Groups . . . . . . . . . . . . . . . . . . . . . 147

Summary . . . . . . . . . . . . . . . . . . . . . . . . . . . . . 147

Additional Resources . . . . . . . . . . . . . . . . . . . . . . . . . 148

CHAPTER 5 Active Directory Security . . . . . . . . . . . . . . . . . 149

Using IP Security . . . . . . . . . . . . . . . . . . . . 150

Understanding IP Security Vulnerabilities $\ldots \ldots 150$

Creating an IPSec Policy . . . . . . . . . . . . . . . . . . . . 152

Managing IPSec from the Command Line . . . . . . . . . . 157

Strengthening TCP/IP Stack Security . . . . . . . . . . . . . . . 161 
Securing Domain Controllers . . . . . . . . . . . . . . . . . . . . 164

Configuring User Rights . . . . . . . . . . . . . . . . . . . 169

Enabling Restricted Groups . . . . . . . . . . . . . . . . . 171

Configuring Authentication Protocols . . . . . . . . . . . 173

Controlling Anonymous Access to Active Directory . . . . . . . . . 175

Creating an Audit Policy . . . . . . . . . . . . . . . . . . . . 176

Monitoring User Activity . . . . . . . . . . . . . . . . . 180

Collecting Auditing Information . . . . . . . . . . . . . . 181

Summary . . . . . . . . . . . . . . . . . . . . . . . . . . . . 184

Additional Resources . . . . . . . . . . . . . . . . . . . . . . . . . 184

CHAPTER 6 Managing Large-Scale Deployments . . . . . . . . . . . 187

Managing Multiple Domains and Forests _ . . . . . . . . . . . . 187

Delegating Authority . . . . . . . . . . . . . . . . . . . 188

Managing Trust Relationships . . . . . . . . . . . . . . . 193

Managing Active Directory Sites . . . . . . . . . . . . . . . . . 202

Configuring Replication . . . . . . . . . . . . . . 205

Using Application Directory Partitions . . . . . . . . . . . . . 212

Troubleshooting Replication Issues . . . . . . . . . . . . . . . 214

Summary . . . . . . . . . . . . . . . . . . . 217

Additional Resources . . . . . . . . . . . . . . . . . . . . . . 218

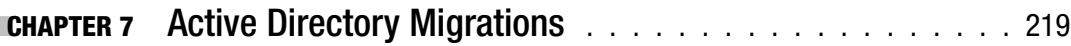

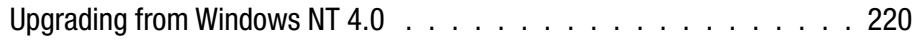

Performing In-Place Upgrades . . . . . . . . . . . . . . . . . . . 221

Restructuring an NT 4.0 Environment . . . . . . . . . . . . . . . . . . 234

Securing the Migration Process . . . . . . . . . . 236

Migrating User Profiles . . . . . . . . . . . . . . . . . 238

Using the Active Directory Migration Tool $\ldots \ldots 239$

Migration Scenarios . . . . . . . . . . . . . . . . . . . . 240

Performing an Interforest Migration . . . . . . . . . . . . . 242

Summary . . . . . . . . . . . . . . . . . . 250

Additional Resources . . . . . . . . . . . . . . . . . . . . . . . 250

CHAPTER 8 Disaster Recovery . . . . . . . . . . . . . . . . . . . . . . 251

Troubleshooting System Startup . . . . . . . . . . . . . . . . 252

Using Alternate Startup Modes . . . . . . . . . . . . . . . 252

Using the Recovery Console . . . . . . . . . . . . . . . . . . 255

Backing Up and Restoring Active Directory . . . . . . . . . . . . 258

Using Windows Backup . . . . . . . . . . . . . . . . 259

Using Automated System Recovery . . . . . . . . . . . . . . 260

Performing Active Directory Restores . . . . . . . . . . . . . 263

Creating a Test Network . . . . . . . . . . . . . . . . . . . . . . . 275

Summary . . . . . . . . . . . . . . . . . . . . . . 280

Additional Resources . . . . . . . . . . . . . . . . . . . . . . . . . 280 
CHAPTER 9 Scripting and Schem(a)ing . . . . . . . . . 281

So What's This Schema Business

(and Why Do I Care)? . . . . . . . . . . . . . . . . . . . . . 282

Using Active Directory Application Mode . . . . . . . . . . . . . 294

A Scripting Primer . . . . . . . . . . . . . . . . . . . . . . . . . . . 299

Getting Started . . . . . . . . . . . . . . . . . . . 299

More Scripting Tricks to Make Your Life Better . . . . . . . . . 310

Summary . . . . . . . . . . . . . . . . . . . . . . 317

Additional Resources . . . . . . . . . . . . . . . . . . . . . . . 317

INDEX . . . . . . . . . . . . . . . . . . . . 319 


\section{About the Author}

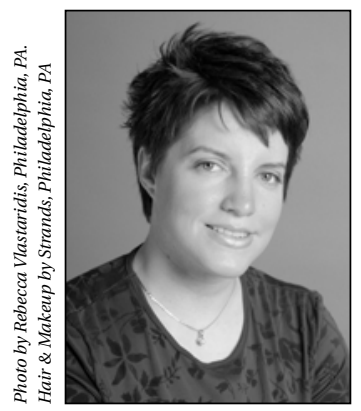

Laura E. Hunter (CISSP, MCSE: Security, MCDBA, Microsoft MVP) is a senior IT specialist with the University of Pennsylvania, where she provides network planning, implementation, and troubleshooting services for various business units and schools within the university. Her specialties include Microsoft Windows 2000 and 2003 design and implementation, troubleshooting, and security topics. Laura has over a decade of experience in the areas of Windows and Novell networking; her previous experience includes a position as the director of computer services for the Salvation Army and as the LAN administrator for a medical supply firm. She is a contributor to the TechTarget family of websites, and to Redmond magazine (formerly Microsoft Certified Professional Magazine).

Laura has previously contributed to the Syngress Windows Server 2003 MCSE/MCSA DVD Guide \& Training System series for exams 70-291/292/293/ $294 / 296 / 297 / 298$ as a DVD presenter, author, and technical reviewer. Laura is a two-time recipient of the prestigious Microsoft "Most Valued Professional" award in the area of Windows Server-Networking. Laura graduated with honors from the University of Pennsylvania and also works as a freelance writer, trainer, speaker, and consultant based in the Philadelphia area. You can reach Laura at laurahcomputing@gmail.com. 


\section{About the Technical Reviewer}

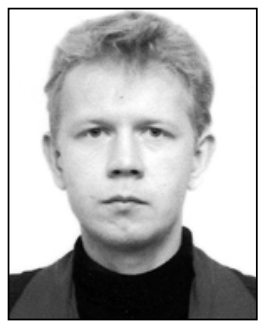

Alexzander Nepomnjashiy is a Microsoft SQL Server database designer for NeoSystems NorthWest-a security services, consulting, and training company.

He has over ten years' experience in the IT field. Currently he is working on several projects that involve the deployment of Microsoft Windows NT Server/Microsoft SQL Server within an enterprise business/financial environment.

His typical role in these projects is extending and improving client's corporate ERP systems to manage retail sales data, predict market changes, and calculate trends for future market situations (using DSS and OLAP tools and techniques).

You can contact him at alexnep@onego.ru. 


\section{Acknowledgments}

project of this scope is never the work of just one individual, and I would like to thank the following people who have been indispensable in nurturing this book through to its final form:

- Everyone at Apress who took the time to make me feel like such a welcome addition to the family: Gary Cornell, Jim Sumser, Beckie Stones, Tina Nielsen, Ellie Fountain, Ami Knox, Julie Miller, Glenn Munlawin, and Jonathan Hassell, along with Alexzander Nepomnjashiy for his outstanding insights as a technical reviewer.

- The wonderful people at Microsoft whom I've engaged with as a part of the MVP program and otherwise: Emily Freet, John Buscher, Eddy Malik, Christopher Corbett, Joseph Davies, Sean O'Driscoll, Susan Leiter, Candice Pedersen, Jan Shanahan, Mark Mortimore, and Steve Riley.

- Members of the Microsoft MVP community who have impacted me both professionally and personally: Steve Friedl, Susan Bradley, Joe Richards, Don Wells, Jeremy Moskowitz, Mark Minasi, Mitch Ruebush, Roger Abell, Thomas Lee, Charles Clarke, Roger Seielstad, Tony Murray, Dèjì Akómöláfé, Robbie Allen, and Ron Chamberlin.

- And last but certainly not least, my family: Carol, Charles, John and Paula Hunter, Stephanie Adams, Wayne Collins, Joey Huff, and Bryan Hopkins. 


\section{Introduction}

ctive Directory Field Guide is predominantly targeted at network administrators and consultants who have some experience with the Windows client and server operating systems. These administrators may fall into one of two groups:

- Readers who have little or no Active Directory experience and who are ready to make the transition from Windows NT 4.0

- Readers who have gained some Active Directory exposure either on the job or through an $\mathrm{AD}$ tutorial, and who are seeking to expand their $\mathrm{AD}$ administration repertoire

This book does not assume that you have any grounding in VBScript, JScript, or any other language commonly used for administrative scripting, though a little background knowledge does no harm. You'll find several examples of these types of scripts throughout the book, as well as a scripting primer at the end of the final chapter.

\section{What Does This Book Cover?}

In structuring this book, I tried to organize things in a logical manner that kept similar tasks and concepts grouped closely together so that you could find them easily. The first two chapters of this book revolve around installing a brand-new Active Directory infrastructure, both installing AD itself as well as the network infrastructure underpinnings that $\mathrm{AD}$ needs to function well. From there, you'll move into a trio of chapters about the kinds of tasks that you'll deal with on a daily basis: administering and securing your Active Directory network. You can take these three chapters in particular as one big chunk - they'll cover most of the skills you'll need to run the day-to-day operations of Active Directory.

The remaining chapters examine different aspects of Active Directory deployment and planning, including managing larger installations and protecting your network against disasters. Over the course of these nine chapters, you'll learn how to install, deploy, configure, and troubleshoot all of the major aspects of Active Directory. Each chapter also closes with a list 
of resources, both print and online, that you can reference for more information on a particular topic.

Here's a summary of the contents of this book:

- Chapter 1 is an introduction to installing Active Directory on a Windows 2000 or Windows Server 2003 computer. This is where we take the "view from 30,000 feet" and look at design considerations such as how your domains and forests should be set up, what your physical and logical design should look like, and how to plan for the necessary hardware capacity for your Active Directory domain controllers. We'll then walk through the process of installing a brand new forest, a new domain tree in an existing forest, a new child domain, and an additional domain controller within an existing domain. We'll also look at ways to automate the installation process through the use of scripts and unattend.txt files.

- Chapter 2 focuses specifically on the network infrastructure components that make Active Directory tick. The most critical of these is DNS, since this is what $\mathrm{AD}$ relies on for client and server name resolution. I'll show you ways to configure DNS zones and servers to optimize name resolution performance on your Active Directory network. We'll then look at some other technologies that are not required for $\mathrm{AD}$ to function, but that you'll probably need to work with at some point nonetheless. This includes DHCP, which is used to automate TCP/IP addressing and configuration for client servers and workstations, as well as WINS, which is used to streamline name resolution for legacy clients and line-of-business applications that still rely on NetBIOS instead of pure DNS.

- Chapters 3 and 4 are all about the day-to-day tasks you'll perform as an Active Directory administrator. This includes creating user and group accounts, managing user profiles and data, and creating login scripts to improve the user experience. In particular, I'll show you ways to automate user creation so that you can create hundreds of Active Directory users at the command line using a simple text file. In Chapter 4, I'll focus specifically on Active Directory Group Policy Objects, which allow you to customize virtually every aspect of your server and client computers from your administrative workstation. This includes using security templates and software restriction policies to protect your network from malicious software or errant users, as well as using software installation settings to deploy line-of-business applications at the touch of a button.

- Chapter 5 focuses on Active Directory security, a topic that has become increasingly important as more and more organizations are acquiring always-on Internet connectivity. We'll look at ways to harden the Windows server operating system and the TCP/IP stack, as well as using IPSec to filter unwanted traffic away from your domain controllers, member servers, and workstations. 
- In Chapter 6, we'll move away from day-to-day tasks and go back up to 30,000 feet to look at managing large-scale Active Directory deployments. In larger environments, you'll probably find yourself in a position where you want to allow local administrators or help desk personnel to take over some of the more common tasks like resetting passwords or unlocking user accounts, and Active Directory gives you much more flexibility in this regard than any of its predecessors. We'll also look at some tasks that are geared specifically towards multisite or multidomain environments, such as setting up trust relationships, site replication, and Global Catalog servers.

- Chapter 7 focuses specifically on performing migrations to Active Directory from earlier versions of the Windows server operating systems, particularly Windows NT 4.0. We'll again start at the high-level planning stages and discuss what you need to do to prepare for a successful (or even an unsuccessful) migration. You'll then get an in-depth look at the Active Directory Migration tool, a free utility that helps to streamline the process of moving users, computers, and other resources from Windows NT 4.0 or Windows 2000 into a new Windows Server 2003 Active Directory environment.

- In Chapter 8, you'll gain some insight into disaster recovery, a crucial topic for any Active Directory administrator. Too many network administrators put off creating a disaster recovery strategy or even a data backup plan until it's too late, after the server has already died (taking your data with it) and you have no good way to get your server's clients back online. To help make sure that this horrible eventuality doesn't happen to you, I'll show you ways to protect your Active Directory database using data backups, as well as ways to troubleshoot a failed server.

- I close out the Active Directory Consultant's Guide with a chapter that has a bit of a split personality: Chapter 9 covers two slightly more advanced topics that you can add to your administrative skillset. We start out by looking at the Active Directory schema, which is the structure that defines how $\mathrm{AD}$ objects and information are created, indexed, and organized throughout your network. This includes how to manage and protect the schema, as well as ways to programmatically extend the schema to customize Active Directory for your business needs. We close things out with a quick introduction to using scripting languages to improve your Active Directory administrative skills. While I don't imagine that I'll turn you into a scripting whiz kid in the space of 15 pages, the final section of this book should at least put you on the path toward scripting excellence. 